\title{
Exponential Growth Of Solutions Of Higher-Order Viscoelastic Wave Equation With Logarithmic Term
}

\author{
Erhan Pişkin ${ }^{1}\left(\mathbb{D}\right.$ and Nazlı Irkıl ${ }^{2 *}$ \\ ${ }^{1}$ Dicle University, Department of Mathematics, Diyarbakır, Turkey, \\ ${ }^{2}$ Mardin Said Nursi Anatolian High School, Mardin, Turkey,
}

Geliş / Received: 24/10/2019, Kabul / Accepted: 21/02/2020

\begin{abstract}
This paper deals with a higher-order viscoelastic wave equation with logarithmic source term. We prove, for suitable conditions, the exponential growth of solutions.

Keywords: Higher-order equation, Growth, Logarithmic nonlinearity.
\end{abstract}

\section{Logaritmik Kaynak Terimli Yüksek Mertebeden Viskoelastik Dalga Denkleminin Çözümlerinin Üstel Büyümesi}

\section{Özet}

$\mathrm{Bu}$ çalışma logaritmik kaynak terimli yüksek mertebeden viskoelastik dalga denklemi ile ilgilidir. Uygun koşullar altında çözümlerin üstel büyümesini ispatladık.

Anahtar Kelimeler: Yüksek mertebeden denklem, Büyüme, Logaritmik terim.

\section{Introduction}

In this paper, we deal with the exponential growth of solutions of the following higherorder viscoelastic wave equation with logarithmic term.

$$
\left\{\begin{array}{l}
u_{t t}+\Delta^{2} u_{t t}+(-\Delta)^{m} u+u \\
-\int_{0}^{t} g(t-s)(-\Delta)^{m} u d s=k u l n|u|, \quad x \in \Omega \times(0, T), \\
u(x, 0)=u_{0}(x), \quad u_{t}(x, 0)=u_{1}(x), \quad x \in \Omega, \\
u(x, t)=\frac{\partial^{i}}{\partial v^{i}} u(x, t)=0, \quad i=1,2, \ldots m-1, x \in \partial \Omega \times(0, T),
\end{array}\right.
$$

where $\Omega \subset R^{3}$ is a bounded domain with smooth boundary $\partial \Omega, v$ is the unit outer normal, $k$ is nonnegative constants to be chosen later and $P=(-\Delta)^{m},(m \in N)$. The kernel $g$ has some conditions to be specified later.

The logarithmic nonlinear source term is related with many branches of physics. Cause of this is interest in it occures naturally in inflation cosmology and supersymmetric field theories, quantum mechanics, nuclear physics (Bialynicki-Birula et al.,1975; Buljan et al., 2003; Martino et al., 2003; Gorka, 2009). A lot of authors studied hyperbolic type equation 
with logarithmic term. (see; Bartkowski et al., 2008; Han, 2013; Messaoudi et al., 2017a and 2017b, Pişkin et al. 2019a and 2019b).

In 1970, the working of Dafermos with viscoelastic term provide a basis to the different papers (Dafermos, 1970). The importance of the viscoelastic properties of materials has been realized because of the rapid developments in rubber and plastic industry. Additionally, viscoelasticity influence part on working of biological phenomena. The other important property of viscoelastic material that return back to its original size after a impact force cut off (Cavalcanti, 2002).

When $m=2$, problem (1) becomes the following

$\left|u_{t}\right|^{\rho} u_{t t}+\Delta^{2} u_{t t}+\Delta^{2} u+u$

$-\int_{0}^{t} g(t-s) \Delta^{2} u d s=k u \ln |u|$

Al-Gharabli et al. investigated the local existence, global existence and stability for problem (2) (Al-Gharabli et al,). Later, they proved the existence and decay results of problem (2) for $\rho=0$ and absence $\Delta^{2} u_{t t}$ term (Al-Gharabli et al., 2019).

Peyravi consider

$u_{t t}-\Delta u+u-\int_{0}^{t} g(t-s) \Delta u d s$

$+h\left(u_{t}\right) u_{t}+|u|^{2} u=u \ln |u|^{k}$

in $\quad \Omega \subset R^{3} \quad$ with $\quad h(s)=k_{0}+k_{1}|s|^{m-1}$ (Peyravi 2018). He studied decay estimate and exponential growth of solutions for problem (3).

Motivated by the above studies, we asked that what results will be obtained if one revises the Laplace operator by other high order viscoelastic term. Additionally, to the best of our knowledge, the growth of solution for the higher-order viscoelastic wave equation with logarithmic source term have not been well studied. Then, we established the exponential growth of the solution for problem (3).

The rest of our work is organized as follows. In section 2, we gave some notations and lemmas which will be used throughout this paper. In section 3, we get suitable conditions for growth of the solutions the problem.

\section{Preliminaries}

In this part, we present some notations and lemmas which will be used throughout this paper. We denote $\|\cdot\|_{2}=\|\cdot\|$ and $\|\cdot\|_{p}$ the usual $L^{2}(\Omega)$ norm and $L^{p}(\Omega)$ norm, respectively. We take by $C$ and $C_{i}(i=$ $1,2, \ldots)$ various positive constants.

Now, we give some important lemmas:

Lemma 1. (Gross, 1975) (Logarithmic Sobolev Inequality). Let $\mathrm{u}$ be any function $u \in$ $H_{0}^{1}(\Omega), \Omega \subset R^{3}$ be a bounded smooth domain and $a>0$ be any number. Then,

$\int_{\Omega} \ln |u| u^{2} d x \leq\left(\frac{3}{4} \ln \frac{4 a}{e}\right)\|u\|^{2}$

$$
+\frac{a}{4}\|\nabla u\|^{2}+\ln \|u\|\|u\|^{2}
$$

Corollary 2. Let $\mathrm{u}$ be any function $u \in$ $H_{0}^{m}(\Omega), \Omega \subset R^{3}$ be a bounded smooth domain and $a>0$ be any number and where $c_{p}$ is the smallest positive number satisfying

$$
\|\nabla u\|^{2} \leq c_{p}\left\|P^{\frac{1}{2} u}\right\|^{2}, \forall u \in H_{0}^{m}(\Omega) .
$$

Then, we obtain 


$$
\begin{aligned}
\int_{\Omega} \ln |u| u^{2} d x & \leq\left(\frac{3}{4} \ln \frac{4 a}{e}\right)\|u\|^{2} \\
& +\frac{c_{p} a}{4}\left\|P^{\frac{1}{2}} u\right\|^{2}+\ln \|u\|\|u\|^{2}
\end{aligned}
$$

Now, we present following assumptions:

(A1) $g: R^{+} \rightarrow R^{+}$is a $C^{1}$ nonincreasing function satisfying

$g(s) \geq 0, \int_{0}^{\infty} g(s) d s<\infty, 1-\int_{0}^{\infty} g(s) d s=l_{0}>0$,

Lemma 3. The energy functional $E(t)$ is decreasing with respect to $t$. Where

$$
\begin{aligned}
& E(t)=\frac{1}{2}\left\|u_{t}\right\|^{2}+\frac{1}{2}\left\|\Delta u_{t}\right\|^{2} \\
& \quad+\frac{1}{2}\left(1-\int_{0}^{t} g(s) d s\right)\left\|P^{\frac{1}{2}} u\right\|^{2} \\
& +\frac{1}{2} g \circ P^{\frac{1}{2}} u+\frac{k+2}{4}\|u\|^{2}-\frac{1}{2} \int_{\Omega} \ln |u|^{k} u^{2} d x .
\end{aligned}
$$

Proof. We multiply both sides of (1) by $u_{t}$ and then integrating over $\Omega$, we have

$$
\begin{aligned}
& \int_{\Omega} u_{t t} u_{t} d x+\int_{\Omega} \Delta_{t t} \Delta u_{t} d x+\int_{\Omega} P^{\frac{1}{2}} u P^{\frac{1}{2}} u_{t} d x \\
& -\int_{\Omega} \int_{0}^{t} g(t-s)(-\Delta)^{m} u d s+\int_{\Omega} u u_{t} d x \\
& =\int_{\Omega} \ln |u|^{k} u u_{t} d x, \\
& \frac{d}{d t}\left(\frac{1}{2}\left\|u_{t}\right\|^{2}\right)+\frac{d}{d t}\left(\frac{1}{2}\left\|\Delta u_{t}\right\|^{2}\right) \\
& +\frac{d}{d t}\left[\frac{1}{2}\left(1-\int_{0}^{t} g(s) d s\right)\left\|P^{\frac{1}{2}} u\right\|^{2}\right] \\
& +\frac{d}{d t}\left(\frac{1}{2} g \circ P^{\frac{1}{2}} u\right)+\frac{d}{d t}\left(\frac{k+2}{4}\|u\|^{2}\right) \\
& -\frac{d}{d t}\left(\frac{1}{2} \int_{\Omega} \ln |u|^{k} u^{2} d x\right) \\
& =\frac{1}{2}\left[g^{\prime} \circ P^{\frac{1}{2}} u-g(t)\left\|P^{\frac{1}{2}} u\right\|^{2}\right], \\
& E^{\prime}(t)=\frac{1}{2}\left[g^{\prime} \circ P^{\frac{1}{2}} u-g(t)\left\|P^{\frac{1}{2}} u\right\|^{2}\right] \leq 0
\end{aligned}
$$

Next, we define the following functional on $H_{0}^{m}(\Omega)$,

$$
\begin{aligned}
I(t) & =\left(1-\int_{0}^{t} g(s) d s\right)\left\|P^{\frac{1}{2}} u\right\|^{2}+g \circ P^{\frac{1}{2}} u \\
& +\|u\|^{2}-\int_{\Omega} \ln |u|^{k} u^{2} d x,
\end{aligned}
$$

$J(t)=\frac{k}{4}\|u\|^{2}+\frac{1}{2} I(t)$,

$E(t)=\frac{1}{2}\left\|u_{t}\right\|^{2}+\frac{1}{2}\left\|\Delta u_{t}\right\|^{2}+J(t)$

where

$$
g \circ P^{\frac{1}{2}} u=\int_{0}^{t} g(t-s)\left\|P^{\frac{1}{2}} u(t)-P^{\frac{1}{2}} u(s)\right\|^{2} d t .
$$

\section{Exponential Growth}

In this part, we will prove exponential growth of solutions to problem (1). Now, we introduce the following

$C_{0}=1-\frac{3 k}{4} \ln \left(\frac{4 a}{e}\right), \gamma^{*}=e^{\frac{2 C_{0}-k}{2 k}}, E_{1}=\frac{k}{4}\left(\gamma^{*}\right)^{2}$

Firstly we suppose that

$$
\text { (A2) } 0<\alpha<\min \left\{\frac{4 l_{0}}{k c_{p}}, \frac{1}{4} e^{\frac{3 k+4}{3 k}}\right\} \text { and } k \geq 1 \text {. }
$$

Theorem 4. Suppose that (A1), (A2) hold. Assume further $\|u(x, 0)\|_{2}>\gamma^{*}$ and $0<E(0)<$ $E_{1}$. Then the solution (1) grow up as an exponential function when $t \rightarrow \infty$.

Now, we give following two lemmas needed our proof for the theorem.

Lemma 5. Suppose that $I(t)<0$ for all $t>0$. Then $\|u(x, t)\|_{2}>\gamma^{*}$ for all $t>0$.

Proof. By using of (9) and Logarithmic Sobolev inequality, we obtain 


$$
\begin{aligned}
J(t) \geq I(t) & \geq\left(1-\int_{0}^{t} g(s) d s\right)\left\|P^{\frac{1}{2}} u\right\|^{2} \\
& +\|u\|^{2}-\int_{\Omega} \ln |u|^{k} u^{2} d x \\
& \geq\left(l_{0}-\frac{k c_{p} \alpha}{4}\right)\left\|P^{\frac{1}{2}} u\right\|^{2} \\
& -\left(1-\frac{3 k}{4} \ln \frac{4 a}{e}-k \ln \|u\|\right)\|u\|^{2} .
\end{aligned}
$$

Using the condition in (A2) and definition of $C_{0}$ in (10), we get

$I(t)>\left(C_{0}-k \ln \|u\|\right)\|u\|^{2}$.

By the negativity of $I(t)$ and using again (A2) we have

$\|u(x, t)\|^{2}>e^{\frac{C_{0}}{k}}>e^{\frac{2 C_{0}-k}{k}}=\gamma^{*}, \forall t \geq 0$.

Lemma 6. Suppose that $\|u(x, 0)\|_{2}>\gamma^{*}$ and $0<E(0)<E_{1}$. Then $I(t)<0$ for all $t>0$.

Proof. We will use contradiction method in our proof. Firstly our aim to see that $I(0)<0$. If not suppose that $I(0) \geq 0$. Then

$E(0) \geq J(0) \geq \frac{k}{4}\|u\|^{2}+\frac{1}{2} I(0) \geq \frac{k}{4}\left(\gamma^{*}\right)^{2}=E_{1}$,

which is impossible. Now suppose that $t_{0}=$ $\inf \{\tau>0: I(\tau)=0\}$. Hence $t_{0}>0$. So, $I(t)<0$ over $\left(0, t_{0}\right)$ and by continuity $I\left(t_{0}\right)=0$. Then, by Lemma 5 we have hence we have $\|u(x, t)\|_{2}>\gamma^{*}$ for all $0<t<t_{0}$ and from the continuity of $t \rightarrow\|u(x, t)\|_{2}^{2}$ we have $\left\|u\left(x, t^{0}\right)\right\|^{2} \geq \gamma^{*}$. Using of (9) we have

$$
\begin{aligned}
E\left(t_{0}\right) & =\frac{1}{2}\left\|u_{t}\left(x, t_{0}\right)\right\|^{2}+\frac{1}{2}\left\|\Delta u_{t}\left(x, t_{0}\right)\right\|^{2}+J\left(t_{0}\right) \\
& >\frac{k}{4}\left\|u\left(x, t_{0}\right)\right\|^{2}+\frac{1}{2} I\left(t_{0}\right) \\
& \geq \frac{k}{4}\left(\gamma^{*}\right)^{2}=E_{1}
\end{aligned}
$$

this is a contradiction.
Remark 7. By Lemma 5 and Lemma 6 we infered that if $\|u(x, 0)\|_{2}>\gamma^{*}$ and $0<E(0)<$ $E_{1}$ then $\|u(x, t)\|^{2}>\gamma^{*}$ for all $t>0$.

Now, we can state proof of the Theorem 4.

Proof. Let us to define

$L(t)=E_{1}+\varepsilon H(t)$,

and

$H(t)=2 \int_{\Omega} u_{t}(t) u(t) d x+2 \int_{\Omega} \Delta^{2} u_{t}(t) \Delta u(t) d x$,

where $\varepsilon>0$ is a sufficiently small number to be determined later.

By differentiating with respect to $t$, we obtain $H^{\prime}(t)=2 \int_{\Omega}\left(u_{t t} u+u_{t}^{2}\right) d x+2 \int_{\Omega}\left(\Delta^{2} u_{t t} u+\Delta^{2} u_{t} u_{t}\right) d x$

$$
\begin{aligned}
& =2 \int_{\Omega}\left(-\Delta^{2} u_{t t}-P u-\int_{0}^{t} g(t-s) P u d s-u+k u \ln |u|\right) u d x \\
& +2 \int_{\Omega} \Delta^{2} u_{t t} u+2\left\|u_{t}\right\|^{2}+2\left\|\Delta u_{t}\right\|^{2}, \\
& =2\left\|u_{t}\right\|^{2}+2\left\|\Delta u_{t}\right\|^{2}-2\|u\|^{2}-2\left\|P^{\frac{1}{2}} u\right\|^{2} \\
& -2 \int_{\Omega} P^{\frac{1}{2}} u \int_{0}^{t} g(t-s) P^{\frac{1}{2}} u d s+2 k \int_{\Omega} u^{2} \ln |u| d x . \quad \text { (17) }
\end{aligned}
$$

By the using of Young's inequality for all $\varepsilon>0$,

$$
\begin{aligned}
& \int_{\Omega} P^{\frac{1}{2}} u \int_{0}^{t} g(t-s) P^{\frac{1}{2}} u d s \leq \frac{\delta}{2}\left\|P^{\frac{1}{2}} u(t)\right\|^{2} \\
& +\frac{1}{2 \delta}\left(\int_{0}^{t} g(s) d s\right) \int_{\Omega} \int_{0}^{t} g(t-s)\left|P^{\frac{1}{2}} u(t)-P^{\frac{1}{2}} u(s)\right|^{2} d s d x \\
& \quad \leq \frac{\delta}{2}\left\|P^{\frac{1}{2}} u(t)\right\|^{2}+\frac{1}{2 \delta}\left(\int_{0}^{t} g(s) d s\right) g \circ P^{\frac{1}{2}} u(t)
\end{aligned}
$$

Insert (18) into (17), we have

$$
\begin{aligned}
H^{\prime}(t) & \geq 2\left\|u_{t}\right\|^{2}-2\left\|P^{\frac{1}{2}} u\right\|^{2}-2\|u\|^{2}+2\left\|\Delta u_{t}\right\|^{2} \\
& -\delta\left\|P^{\frac{1}{2}} u(t)\right\|^{2}-\frac{1}{\delta}\left(\int_{0}^{t} g(s) d s\right) g \circ P^{\frac{1}{2}} u(t)
\end{aligned}
$$

$$
+2 k \int_{\Omega} u^{2} \ln |u| d x .
$$




$$
\begin{aligned}
\geq & 2\left\|u_{t}\right\|^{2}+2\left\|\Delta u_{t}\right\|^{2}-(2+\delta)\left\|\frac{1}{P^{2}} u\right\|^{2}-2\|u\|^{2} \\
& -\frac{1}{\delta}\left(\int_{0}^{t} g(s) d s\right) g \circ P^{\frac{1}{2}} u(t)+2 k \int_{\Omega} u^{2} l n|u| d x .
\end{aligned}
$$

Adding and subtracting $\eta I(t)$ into (19), we have

$$
\begin{aligned}
H^{\prime}(t) & \geq 2\left\|u_{t}\right\|^{2}+2\left\|\Delta u_{t}\right\|^{2}+\left(\eta-\frac{1}{\delta}\left(\int_{0}^{t} g(s) d s\right)\right) g \circ P^{\frac{1}{2}} u(t) \\
& +[\eta-2]\|u\|^{2}+k(2-\eta) \int_{\Omega} u^{2} \ln |u| d x . \\
& +\left[\eta\left(1-\int_{0}^{t} g(s) d s\right)-(2+\delta)\right]\left\|P^{\frac{1}{2}} u\right\|^{2}-\eta I(t), \quad \text { (20) }
\end{aligned}
$$

where $\eta>2$. For estimating the fifth term (20), we use Corollary 2, we obtain

$$
\begin{aligned}
\int_{\Omega} \ln |u| u^{2} d x & \leq\left(\frac{3}{4} \ln \frac{4 a}{e}\right)\|u\|^{2} \\
& +\frac{c_{p} a}{4}\left\|P^{\frac{1}{2}} u\right\|^{2}+\ln \|u\|\|u\|^{2}
\end{aligned}
$$

By the combining (20) and (21) we have

$$
\begin{aligned}
& H^{\prime}(t) \geq 2\left\|u_{t}\right\|^{2}+2\left\|\Delta u_{t}\right\|^{2} \\
& \quad+\left(\eta-\frac{1}{\delta}\left(\int_{0}^{t} g(s) d s\right)\right) g \circ P^{\frac{1}{2}} u(t)-\eta I(t) \\
& +k(2-\eta)\left[\left(\frac{3}{4} \ln \frac{4 a}{e}\right)\|u\|^{2}+\frac{c_{p} a}{4}\left\|P^{\frac{1}{2}} u\right\|^{2}+\ln \|u\|\|u\|^{2}\right] \\
& +[\eta-2]\|u\|^{2}+\left[\eta\left(1-\int_{0}^{t} g(s) d s\right)-(2+\delta)\right]\left\|P^{\frac{1}{2}} u\right\|^{2} \\
& \geq 2\left\|u_{t}\right\|^{2}+2\left\|\Delta u_{t}\right\|^{2}+\left(\eta+\frac{1}{\delta}-\frac{l_{0}}{\delta}\right) g \circ P^{\frac{1}{2}} u(t) \\
& +(2-\eta)\left[\left(\frac{3 k}{4} \ln \frac{4 a}{e}+k \ln \|u\|\right)-1\right]\|u\|_{2}^{2} \\
& {\left[\eta l_{0}-(2+\delta)+k(2-\eta) \frac{c_{p} \alpha}{4}\right]\left\|P^{\frac{1}{2}} u\right\|^{2}-\eta I(t)}
\end{aligned}
$$

Using the condition in (A2) and definition of $C_{0}$ in (10), we can select $\eta$ large so that

$\eta>\max \left\{2, \frac{l_{0}-1}{\delta}, \frac{8-8 k+4 \delta}{4 l_{0}-k c_{p} \alpha}\right\}$

Consequently because of Lemma 6, for some $c_{i}>0 .(i=0,1,2 \ldots)$, we can write (22)

$$
\begin{aligned}
H^{\prime}(t) & \geq 2\left\|u_{t}\right\|^{2}+2\left\|\Delta u_{t}\right\|^{2}+c_{0}\|u\|^{2} \\
& +c_{1}\left(g \circ P^{\frac{1}{2}} u\right)(t)+c_{2}\left\|P^{\frac{1}{2}} u\right\|^{2} .
\end{aligned}
$$

Thus, we have

$$
L^{\prime}(t)=\varepsilon H^{\prime}(t)>0 .
$$

Hence, $L$ is an increasing function on $(0, \infty)$ and taking $\varepsilon$ small enough such that $L(0)>0$ so that $L(t)>0$ for all $t \geq 0$.

On the other hand, by using the Hölder, Young inequalities and Poincare inequalities in (15) and then by Remark 7 and (23) we obtain

$$
\begin{aligned}
L(t) & \leq \frac{k}{4}\left(\gamma^{*}\right)^{2}+2 \varepsilon\|u\|^{2} \\
& +\varepsilon\left\|u_{t}\right\|^{2}+2 \varepsilon\|\Delta u\|^{2}+\varepsilon\left\|\Delta u_{t}\right\|^{2} \\
& <\frac{k}{4}\|u\|^{2}+2 \varepsilon\|u\|^{2}+\varepsilon\left\|u_{t}\right\|^{2} \\
& +2 \varepsilon\|\Delta u\|^{2}+\varepsilon\left\|\Delta u_{t}\right\|^{2} \\
& <\left(\frac{k}{4}+2 \varepsilon\right)\|u\|^{2}+\varepsilon\left\|u_{t}\right\|^{2} \\
& +2 \varepsilon\left\|P^{\frac{1}{2}} u\right\|^{2}+\varepsilon\left\|\Delta u_{t}\right\|^{2} \\
& <\vartheta(\varepsilon, k) L^{\prime}(t) .
\end{aligned}
$$

Integrate (24), yields

$$
L(t)>e^{\frac{t}{\vartheta(\varepsilon, k)}} L(0) .
$$

This completes the our proof.

\section{References}

Al-Gharabli, M.M., Guesmia A. and Messaoudi, S.A., (2020), Well posedness and asymptotic stability results for a viscoelastic plate equation with a logarithmic nonlinearity, Applicable Analysis, 99(1) 50-74.

Al-Gharabli, M.M., Guesmia A. and Messaoudi, S.A., (2019), Existence and a general decay results for a viscoelastic plate 
equation with a logarithmic nonlinearity, 18(1) 159-180.

Bartkowski, K. and Gorka, P., (2008), Onedimensional Klein--Gordon equation with logarithmic nonlinearities, J. Phys. A., 41(35) $1-11$.

Bialynicki-Birula, I. and Mycielski, J., (1975), Wave equations with logarithmic nonlinearities, Bull. Acad. Polon. Sci. Ser. Sci. Math. Astron. Phys., 23(4) 461-466.

Buljan, H., Siber,,A, Soljacic, M., Schwartz, T., M.,Segev, D. N., Christodoulides, (2003), Incoherent white light solitons in logarithmically saturable noninstantaneous nonlinear media, Phys. Rev., E (3) 68.

Cavalcanti, M., Cavalcanti, V.N.D. and Soriano, J.A., (2002), Exponential decay for the solution of semi linear viscoelastic wave equations with localized damping, Electron. J. Differ. Equ. 2002, 1-14.

Dafermos, C., (1970), Asypmtotic stability in viscoelasticity, Arch. Ration. Mech. Anal., 37, 297-308.

Martino, D., Falanga, M., Godano,C. and Lauro, G., (2003), Logarithmic Schrödingerlike equation as a model for magma transport, Europhys. Lett., 63(3), 472-475.

Gorka, P., (2009), Logarithmic Klein-Gordon equation, Acta Phys. Pol. B 40(1), 5966.

Gross, L., (1975), Logarithmic Sobolev inequalities, Amer. J. Math. 97(4), 1061-1083.

Han, X.S., (2013), Global existence of weak solutions for a logarithmic wave equation arising from Q-ball dynamics, Bull. Korean Math. Soc. 50(1), 275--283.
Messaoudi, S.A. and Al-Gharabli, (2017), Existence and a general decay result for a plate equation with nonlinear damping and a logarithmic source term, Journal of Evolution Equations, 18(1),105-125.

Messaoudi, S.A. and Al-Gharabli, (2017), The existence and the asymptotic behavior of a plate equation with frictional damping and a logarithmic source term, J. Math. Anal. Appl., 454, 1114-1128.

Peyravi, A., (2018), General stability and exponential growth for a class of semi-linear wave equations with logarithmic source and memory terms., Appl. Math. Optim.,1-17.

E. Pişkin and N. Irkı1, (2019), Mathematical Behavior of Solutions of Fourth-Order Hyperbolic Equation with Logarithmic Source Term, CPOST, 2(1), 27-36.

E. Pişkin and N. Irk1l, (2019), Well-posedness results for a sixth-order logarithmic Boussinesq equation, Filomat, 33(13), 39854000. 